\title{
Diabetes en pacientes con infección de piel y partes blandas internados en el Hospital de Clínicas
}

\section{Diabetes in patients with skin and soft tissue infection admitted to Hospital de Clínicas}

Víctor Daniel Giménez Ortigoza ${ }^{1}$; Pablo Ramón Torres ${ }^{1}$

\section{RESUMEN}

Introducción: La frecuencia de diabetes mellitus en el país es alrededor del $13 \%$,en general las infecciones de piel y partes blandas en general presentan una evolución desfavorable en este tipo de pacientes.

Objetivos: Determinar la frecuencia de diabetes en pacientes internados con infección de piel y partes blandas. Metodología: diseño observacional con componentes analíticos de corte transversal, que incluyó a 236 pacientes adultos internados en el Servicio de Clínica Médica del Hospital de Clinicas ( Paraguay) durante 2016 y 2019. Resultados: la edad media fue $52 \pm 11$ años, hubo predominio de sexo masculino (58\%). Las entidades más diagnosticadas fueron celulitis ( $50 \%$ ), erisipela(14\%), La comorbilidad más frecuente fue la diabetes mellitus en un 39\%, de los cuales el $63 \%$ no sabía que tenía la enfermedad. El Staphylococcus aureus fue el germen más frecuentemente aislado en los cultivos ( $55 \%$ ) y se encontró asociación significativa entre cultivo positivo para Staphylococcus aureus vs diabetes mellitus. Conclusión: la celulitis fue la infección de piel y partes blandas más frecuente, la mayoría causada por Staphylococcus aureus. La diabetes mellitus fue la comorbilidad más frecuente.

Palabras clave: celulitis, diabetes mellitus, fascitis necrotizante, Staphylococcus aureus. 


\section{ABSTRACT}

Introduction: The frequency of diabetes mellitus in the country is around $13 \%$, in general skin and soft tissue infections in general present an unfavorable evolution in this type of patients.

Objectives: To determine the frequency of diabetes in hospitalized patients with skin and soft tissue infections. Methodology: observational design with analytical cross-sectional components, which included 236 adult patients hospitalized in the Medical Clinic Service of the Hospital de Clinicas (Paraguay) during 2016 and 2019. Results: the mean age was $52 \pm 11$ years, there was a predominance of males $(58 \%)$. The most diagnosed entities were cellulite (50\%), erysipelas (14\%). The most frequent comorbidity was diabetes mellitus in $39 \%$ of which $63 \%$ of them did not know they had the disease. Staphylococcus aureus was the most frequently isolated germ in cultures (55\%) and a significant association was found between positive cultures for Staphylococcus aureus vs diabetes mellitus. Conclusion: cellulite was the most frequent skin and soft tissue infection, the majority caused by Staphylococcus aureus. Diabetes mellitus was the most frequent comorbidity.

Keywords: Cellulitis, diabetes melllitus, Fasciitis Necrotizing, Staphylococcus aureus. 


\section{INTRODUCCIÓN}

Las infecciones de piel y partes blandas (IPPB) son motivos frecuentes de consulta y causan un importante uso de antibióticos a nivel mundial. Las IPPB presentan formas clínicas muy variables desde procesos limitados a grandes infecciones sistémicas. Se han clasificado las IPPB en 2 categorías: infecciones superficiales o simples (impétigo, erisipela y celulitis) e infecciones profundas, complicadas o necrosantes (fascitis y mionecrosis) (1).

La frecuencia de diabetes mellitus (DM) en el país es alrededor del $13 \%$, en general las infecciones en las personas con $\mathrm{dm}$ puede ser más tórpida, presentan mayor riesgo de complicaciones y mortalidad, generan mayor impacto económico en el sistema de salud y estancia hospitalaria mayor $(2,3)$.

\section{MÉTODO}

Se realizó un estudio observacional descriptivo de corte transversal con componentes analíticos. El muestreo fue no probabilístico de casos consecutivos de pacientes internados en el Servicio de Medicina Interna del Hospital de Clínicas - Facultad de Ciencias Médicas. (San Lorenzo, Paraguay) durante enero del 2016 a diciembre 2019.

\section{Criterios de inclusión:}

Pacientes internados con diagnostico al ingreso hospitalario de IPPB por clínica o imágenes que acudieron al consultorio externo o urgencias, independientemente de si fueron superficiales o profundas al momento del ingreso.

\section{Pacientes Mayores de 18 años.}

Pacientes con o sin intervención quirúrgica a causa de las IPPB

\section{Criterios de exclusión:}

Pacientes con diagnóstico de VIH, leucemias y otras neoplasias.

Ulceras de decúbito, lesiones por insuficiencia arterial o isquemia aguda.
No está definido si las IPPB son más frecuentes en diabéticos o en la población general, en general los estudios presentan resultados discordantes. Las IPPB afectan principalmente a pacientes con enfermedades de base predisponentes, la diabetes junto con la insuficiencia cardíaca es a comorbilidad más frecuentemente asociada (4).

El aislamiento microbiológico más frecuente en las IPPB es Staphylococcus aureus, sobre todo en las infecciones superficiales (4). La localización de las extremidades inferiores es actualmente más frecuente. Las complicaciones locales y generales que se informan con frecuencia son abscesos, necrosis superficial o trombosis venosa profunda (5).

El objetivo de esta investigacion fue determinar la frecuencia de DM en pacientes con IPPB.

IPPB desarrolladas durante la internación, secundarias a accidentes automovilísticos o motocicleta.

Quemaduras o lesiones por agentes químicos.

Historias clínicas incompletas o dañadas

Metodología: se revisaron las historias clínicas de los pacientes internados en el Servicio de Medicina con diagnóstico de IPPB al ingreso, una vez seleccionadas las historias se completó las planillas prediseñadas para la recolección de datos y se procedió al análisis de los mismos.

\section{Variables de estudio:}

Cuantitativa: edad, días de signos y síntomas.

Cualitativa: sexo, cultivos, tipo de IPPB (superficiales o profunda), comorbilidades (diabetes mellitus, hipertensión arterial, obesidad, uso de corticoides, etilismo, insuficiencia cardiaca), requerimiento de unidad de cuidados intensivos y óbito, antecedente de consmo de antibióticos. 


\section{Definiciones operacionales}

Criterios diagnóstico para Diabetes ALAD 2019(6).

-Glucosa en ayuno $\geq 126 \mathrm{mg} / \mathrm{dL}$ (no haber tenido ingesta calórica en las últimas 8 horas).

-Glucosa plasmática a las 2 horas de $\geq 200 \mathrm{mg} / \mathrm{dL}$ durante una prueba oral de tolerancia a la glucosa.

-Hemoglobina glucosilada (A1C) $\geq 6.5 \%$.

-Paciente con síntomas clásicos de hiperglicemia o crisis hiperglucémica con una glucosa al azar $\geq 200 \mathrm{mg} / \mathrm{dL}$.

\section{Clasificación de la presión arterial 2017 (7). \\ Categoría \\ Normal \\ Elevada \\ Hipertensión estadio 1 \\ Hipertensión estadio 2 \\ Cifras de presión arterial $(\mathrm{mmHg})$

$$
<120 / 80
$$$$
120-129 /<80
$$$$
130-139 / 80-89
$$$$
\geq 140 / 90
$$

\section{Obesidad y síndrome metabólico}

Se usó para el diagnóstico del SM la presencia de al menos tres de los cinco criterios propuestos por el reciente consenso de la Federación Internacional de DiaBetes y de la Asociación Internacional para el Estudio de la Obesidad (7).

\section{Clasificación de la presión arterial 2017 (7).}

-Circunferencia de cintura elevada de acuerdo a la población

-Triglicéridos elevados de más de $150 \mathrm{mg} / \mathrm{dl}$, o recibir tratamiento

- HDL menor de $40 \mathrm{mg} / \mathrm{dl}$ en hombres y menor de $50 \mathrm{mg} / \mathrm{dl}$ en mujeres.

-Tensión arterial alta normal o en rango de hipertensión (TAS 130 mmHg y/o TAD

$85 \mathrm{mmHg}$ o recibir tratamiento.

-Glucosa en ayunas mayor de $100 \mathrm{mg} / \mathrm{dl}$ o recibir tratamiento

El perímetro abdominal se considera normal al ser menor de $90 \mathrm{cms}$ para los hombres y menor de $80 \mathrm{cms}$ para las mujeres.

\section{Calculo del tamaño Muestral:}

Se tuvo en cuenta el libro de hulley (8) para el cálculo, para una frecuencia estimada de diabéticos en pacientes con IPPB de 33\%(9) (p) con un nivel de confianza del 95\%, Z: valor correspondiente a la distribución de gauss, $\mathrm{z} \alpha=0.05=1.96$, q: $1-$ $\mathrm{p}$, i: error que se prevé cometer del $5 \%$.E1 tamaño de la muestra calculada fue de 229 participantes.

\section{Gestión y análisis de datos.}

Las variables se registraron en planillas electrónicas Excel. Las cualitativas de expresarán en frecuencias y porcentajes, las cuantitativas en medias y desvío estándar. Para las asociaciones se utilizó el programa Epi info y para las asociaciones chi cuadrado (x2).

Aspectos éticos: Se procedió a análisis de historias clínicas con autorización de Jefe de Servicio y se respetaron los principios de confidencialidad. 


\section{RESULTADOS}

Se incluyeron un total de 236 sujetos, La mayor parte de los casos pertenecen al con predominio de sexo masculino (58\%). grupo etario mayor a 50 años (Gráfico 1).

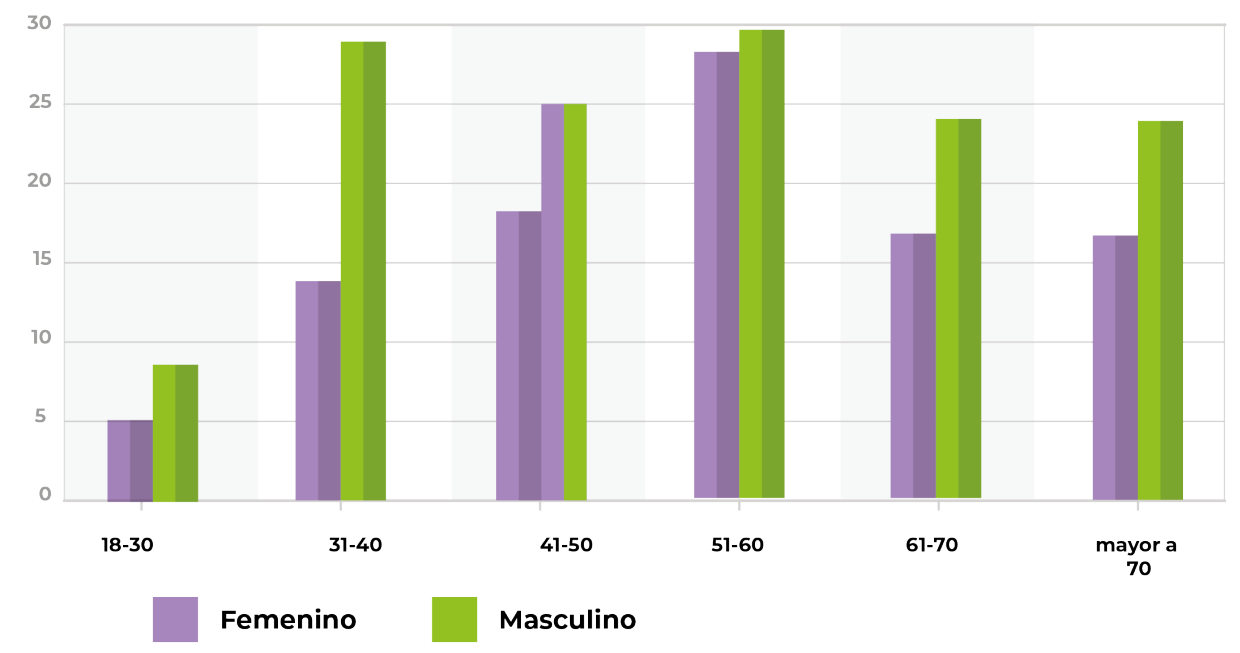

Gráfico 1: Pacientes con IPPB internados en el Hospital de Clínicas según edad y sexo. n: 236

Las entidades más diagnosticadas fue- das de la fascitis (13\%) (Gráfico 2). ron celulitis $(50 \%)$, erisipela $(14 \%)$, segui-

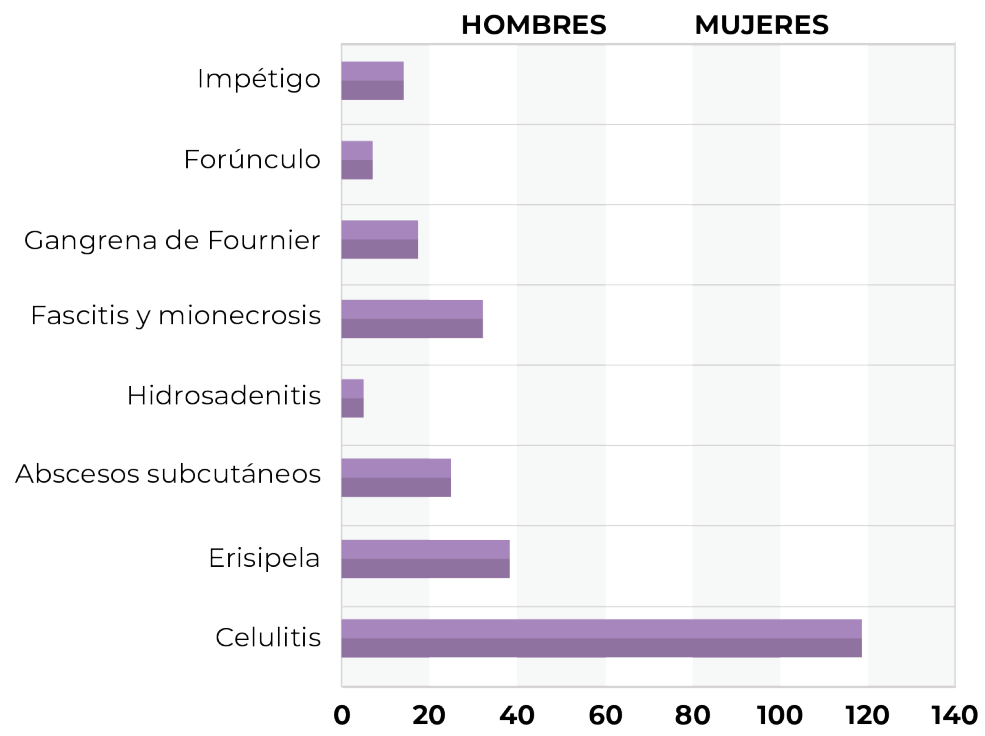

Gráfico 2: Distribución porcentual de las IPPB en pacientes internados en Clínica Médica del Hospital de Clínicas. N: 236 
Las infecciones se localizaron mayoritariamente en las extremidades inferiores 144 casos (61 \%) y en las superiores en $40(17 \%)$, seguidas de genitales y glúteos en $26(11 \%)$. En 44\% de los episodios se registró el antecedente de haber recibido antibioterapia previa por cualquier moti- vo en los últimos 6 meses, principalmente aminopenicilinas con inhibidores de betalactamasas en $31,6 \%$ ocasiones.

La comorbilidad más frecuente fue la DM en un 39\%( Tabla 1) de los cuales el $63 \%$ de los mismos no sabía que tenía la enfermedad.

Tabla 2: Frecuencia de comorbilidades de pacientes con infección de piel y partes blandas. $n: 236$

\begin{tabular}{ll} 
Comorbilidades & Frecuencia \\
\hline Diabetes mellitus & $92(39 \%)$ \\
Síndrome metabólico & $87(33 \%)$ \\
Insuficiencia cardíaca & $40(16,8 \%)$ \\
Hepatopatía crónica & $26(10 \%)$ \\
Hipertensión arterial & $69(28 \%)$ \\
Enfermedad renal crónica & $24(9 \%)$ \\
Conectivopatía & $16(1,6 \%)$
\end{tabular}

El Staphylococcus aureus fue el gérmen más frecuentemente aislado en los cultivos ( $38 \%$ ), seguido de la Pseudomonas aeru- ginosa en un $11 \%$. Requirieron ingreso a unidad de cuidados intensivos el $13 \%$ y falleció el $8 \%$.

\begin{tabular}{|lll|}
\hline Aislamiento microbiológico & $\begin{array}{c}\text { Cultivo foco } \\
(\mathbf{\%}) \mathbf{n}=\mathbf{1 4 5}\end{array}$ & $\begin{array}{c}\text { Hemocultivo } \\
(\mathbf{\%}) \mathbf{n}=\mathbf{1 5 5}\end{array}$ \\
\hline Staphylococcus aureus & $55(38)$ & $29(18)$ \\
Streptococcus pyogenes & $10(8)$ & $7(4,5)$ \\
Pseudomonas aeruginosa & $17(11)$ & $10(6)$ \\
Enterococcus spp. & $6(3,5)$ & $8(5)$ \\
Staphylococcus coagulasa negativo & $12(11)$ & $9(5,8)$ \\
Streptococcus grupo viridans & $10(8)$ & $5(3)$ \\
Enterococcus spp. & $2(1)$ & $1(0,6)$ \\
Escherichia coli & $15(10)$ & $15(9)$ \\
Negativos & $18(12)$ & $71(45)$
\end{tabular}

Se encontró un retardo en la consulta inicial con un promedio $4 \pm 1,5$ días y no se encontró asociación significativa entre ingreso a cuidados intensivo vs diabetes mellitus ( $\mathrm{p}$ 0,23), si se encontró asociación significativa entre cultivo positivo para Staphylococcus aureus vs diabetes ( $p$ $0,013)$. 


\section{DISCUSIÓN}

El estudio aquí presentado tuvo limitaciones, no se tuvo en cuenta el tratamiento antibiótico de la IPPB durante la internación y tampoco se categorizo a los pacientes según estado de patologías de base. Debemos resaltar que en nuestro servicio no ingresan pacientes con requerimiento de desbridamiento inicial por lo que este dato podría contribuir a sesgos.

La frecuencia de DM en pacientes con IPPB fue ligeramente superior en nuestro estudio teniendo en cuenta estudios similares hechos en España (4) alrededor del $30 \%$ y muy superior a los de USA (24\%) (9), lo más llamativo y una de los principales motivos por lo que realizamos esta investigación fue conocer que el $63 \%$ del total de diabéticos no conocía ser portador de dicha enfermedad, lo cual es un dato muy preocupante.
La asociación entre la DM y las infecciones de piel y partes blandas en bien conocida incluso empeora el pronóstico y presentan mayor tasa de utilización de antibióticos $(10,11,12)$, además de que el $77 \%$ de los pacientes en estudio presentaron algún tipo de comorbilidad destacando al síndrome metabólico y la insuficiencia.

El Staphylococcus aureus fue el germen más frecuentemente aislado (38 \%) coincidente con literaturas internacional (13-15), se encontró asociación significativa entre dicho germen y la presencia de diabetes en los pacientes ( $p$ 0,013).

Al igual que los estudios previos $(4,9)$, la mayoría de los sujetos retardaron la consulta inicial $4 \pm 1,5$ días. No se encontró asociación significativa entre ingreso a cuidados intensivo vs diabetes mellitus ( $\mathrm{p} 0,23)$.

\section{CONCLUSIONES}

El Ministerio de Salud y Bienestar Social debe tratar el problema de la diabetes con urgencia ya que existe una gran cantidad de portadores que no saben que padecen dicha enfermedad como lo demuestra nuestra investigación, es bien conocido

que el mal control glicémico predispone a IPPB así como las lesiones en piel. Se concluye igualmente que la celulitis fue la IPPB más frecuente, la mayoría causada por Staphylococcus aureus. La diabetes mellitus fue la comorbilidad más frecuente.

\section{REFERENCIAS}

1. Peralta $\mathrm{R}$, Torres de Taboada E. Infecciones de piel y partes blandas. Rev Virtual la Soc Paraguaya Med Interna [Internet]. 2017 [cited 2020 Jul 8];4(2):1926. Available from: http://scielo.iics. una.py/scielo.php?script $=$ sci_arttext\&pid=S2312-38932017000200019\&1n$\mathrm{g}=\mathrm{en} \& \mathrm{nrm}=\mathrm{iso \& t} \operatorname{lng}=\mathrm{es}$

2. López-Simarro F, Redondo Margüello $\mathrm{E}$, Mediavilla Bravo JJ, Soriano
Llora T, Iturralde Iriso J, Hormigo Pozo A. Prevention and treatment of infectious diseases in diabetic patients. Semergen [Internet]. 2019 Mar 1 [cited 2020 Jul 8];45(2):117-27. Available from: https:// pubmed.ncbi.nlm.nih.gov/30580897/

3. Chaves G, Brítez N, Maciel V, Klinkhof A, Mereles D. Investigación original / Original research [Internet]. Vol. 38, Revista Panamericana de Salud Pú- 
blica. Organización Panamericana de la Salud; 2015 [cited 2020 Jul 8]. Available from: http://clinicaltrials.gov/

4. Raya-Cruz M, Ferullo I, Arrizabalaga-Asenjo M, Nadal-Nadal A, Díaz-Antolín MP, Garau-Colom M, et al. Infecciones de piel y partes blandas en pacientes hospitalizados: factores epidemiológicos, microbiológicos, clínicos y pronósticos. Enferm Infecc Microbiol Clin. 2014 Mar 1;32(3):152-9.

5. Pitché PV, Saka B, Diatta AB, Faye O, Diané BF, Sangaré A, et al. Risk factors associated with abscess formation among patient with leg erysipelas (cellulitis) in sub-Saharan Africa: A multicenter study. BMC Dermatol [Internet]. 2015 Dec 15 [cited 2020 Jul 8];15(1):18. Available from: http://www.biomedcentral. com/1471-5945/15/18

6. Directriz: Estándares ADA 2020 de atención médica en diabetes $\mid$ Noticias y perspectivas médicas gratuitas [Internet]. [cited 2020 Jul 8]. Available from: https:// www.linksmedicus.com/news/guideline-ada-2020-standards-medical-care-diabetes/

7. Rubio-Guerra AF. New guides of American College of Cardiology/ American Heart Association Hypertension for the treatment of high blood pressure. A jump in the right direction? Med Interna Mex [Internet]. 2018 Mar 1 [cited 2020 Jul 8];34(2):299-303. Available from: http://www.scielo.org. $\mathrm{mx} / \mathrm{scielo}$.php? script $=$ sci_arttext\&pi$\mathrm{d}=\mathrm{S} 0186-48662018000200011 \& 1 \mathrm{n}$ $\mathrm{g}=\mathrm{es} \& \mathrm{nrm}=$ iso\&tlng=es

8. Hulley SB, Cummings SR, Browner WS, M.D., Grady DG, Newman TB. Diseño de Las Investigaciones Clinicas. 4a Edición. [Internet]. Lippincott Williams \& Wilkins; 2016 [cited 2020 May 27]. 417 p.
Available from: http://books.google.com/ books?id=ovFmJWAACAAJ\&pgis $=1$.

9. Talan DA, Salhi BA, Moran GJ, Mower WR, Hsieh YH, Krishnadasan A, et al. Factors associated with decision to hospitalize emergency department patients with skin and soft tissue infection. West $\mathrm{J}$ Emerg Med [Internet]. 2015 [cited 2020 Jul 8];16(1):89-97. Available from: https://escholarship.org/uc/item/1405j185

10. Abu-Ashour W, Twells LK, Valcour JE, Gamble JM. Diabetes and the occurrence of infection in primary care: A matched cohort study. BMC Infect Dis [Internet]. 2018 Feb 5 [cited 2020 Jul 8];18(1):67. Available from: https:// bmcinfectdis.biomedcentral.com/articles/10.1186/s12879-018-2975-2

11. Carey IM, Critchley JA, Dewilde S, Harris T, Hosking FJ, Cook DG. Risk of infection in type 1 and type 2 diabetes compared with the general population: A matched cohort study. Diabetes Care [Internet]. 2018 Mar 1 [cited 2020 Jul 8];41(3):513-21. Available from: https:// doi.org/10.2337/dc17-2131

12. Mor A, Berencsi $K$, Nielsen JS, Rungby J, Friborg S, Brandslund I, et al. Clinical Infectious Diseases Rates of Community-based Antibiotic Prescriptions and Hospital-treated Infections in Individuals With and Without Type 2 Diabetes: A Danish Nationwide. 2016 [cited 2020 Jul 8]; Available from: https://academic.oup.com/ cid/article-abstract/63/4/501/2595092

13. Martin ET, Kaye KS, Knott C, Nguyen H, Santarossa M, Evans R, et al. Diabetes and risk of surgical site infection: A systematic review and meta-analysis. Infect Control Hosp Epidemiol [Internet]. 2016 [cited 2020 Jul 8];37(1):88-99. Available from: https://pubmed.ncbi.nlm.nih. gov $/ 26503187 /$ 
14. Wiseman JT, Fernandes-Taylor S, Barnes ML, Saunders RS, Saha S, Havlena $\mathrm{J}$, et al. Predictors of surgical site infection after hospital discharge in patients undergoing major vascular surgery Presented at the 2015 Vascular Annual Meeting of the Society for Vascular Surgery, Chicago, Ill, June 17-20, 2015. In: Journal of Vascular Surgery [Internet]. Mosby Inc.; 2015 [cited 2020 Jul 8]. p. 1023-1031.e5. Available from: https://pubmed.ncbi.nlm.nih. gov/26143662/
15. Tascini C, Piaggesi A, Tagliaferri E, Iacopi E, Fondelli S, Tedeschi A, et al. Microbiology at first visit of moderate-to-severe diabetic foot infection with antimicrobial activity and a survey of quinolone monotherapy. Diabetes Res Clin Pract [Internet]. 2011 Oct [cited $2020 \mathrm{Jul}$ 8];94(1):133-9. Available from: https:// pubmed.ncbi.nlm.nih.gov/21824673/ 